\title{
Trajectory Planning of Redundant Planar Mechanisms for Reducing Task Completion Duration
}

\author{
Emre Uzunoğlu ${ }^{1}$, Mehmet İsmet Can Dede ${ }^{1}$, Gökhan Kiper ${ }^{1}$, Ercan Mastar ${ }^{2}$, \\ Tayfun Sığırtmaç ${ }^{2}$ \\ ${ }^{1}$ Department of Mechanical Engineering, İzmir Institute of Technology, 35430 İzmir, Turkey \\ emreuzunoglu@iyte.edu.tr, candede@iyte.edu.tr, gokhankiper@iyte.edu.tr \\ ${ }^{2}$ Coşkunöz Metal Form Endüstri ve Ticaret A.Ş., R\&D Department, 16159, Bursa, Turkey \\ emastar@coskunoz.com.tr, tsigirtmac@coskunoz.com.tr
}

\begin{abstract}
In the industry there is always a demand to shorten the task completion durations in order to maximize the efficiency of the operation. This work aims to provide a solution to minimize the task completion duration for planar tasks by introducing kinematic redundancy. An example setting of a redundant planar mechanism is considered and an algorithm developed for resolving redundancy order to minimize task completion duration is discussed based on this mechanism.
\end{abstract}

Keywords: trajectory planning, redundant manipulator, redundancy resolution

\section{Introduction}

Kinematically redundant manipulators have numerous advantages over the sufficient manipulators since they can provide the task designer with infinite number of solutions that can achieve the same primary task. Self-motion of the redundant manipulator, which has no effect on primary task (Nakamura, 1991), is used to achieve subtasks (e.g. singularity avoidance, minimizing total joint motion). Redundancy resolution algorithms that allow the ability to optimize for various criteria using subtask controllers have been proposed in literature. Redundancy resolution in velocity making use of pseudo-inverse approaches has been widely used in various subtask controls such as singularity avoidance (Yoshikawa, 1984), joint velocity minimization (Seraji, 1991), obstacle avoidance (Chen et al., 2002), mechanical joint limit avoidance (Tatlıcıoğlu et al., 2009), and manipulability (Maaroof et al., 2012). In addition, different redundancy resolution approaches have been proposed including in velocity level (Rajiv et al., 1991) and in acceleration level (Wang et al., 2010). Examples presented above address optimization for var- 
ious sub-tasks. However, in this work, our focus is on reducing the total task completion duration with maximum allowable accelerations.

The motivation of this paper is to use the advantage of extra degrees of freedom (DoF) of redundant manipulators to develop trajectory generation algorithm to achieve higher acceleration motions in global task space in order to reduce the operation time. Shortening the operation time as much as possible is crucial since it will increase the productivity of the machine. As a fact, in many industrial and robotic applications that include sharp curves in their paths, robot's acceleration performance is required to be as high as possible not to lose much time at the curves. However, in industrial settings, where the manipulators have large workspace, higher accelerations at the tip of the manipulator will call for higher force/torque demands from the actuators of the manipulator. Nevertheless, mechanisms that have higher inertia cannot achieve high acceleration motions since it results in poor precision and may create physical damage in the mechanism due to high amplitude vibrations. Therefore, there are limitations for maximum allowable accelerations for high inertia manipulators. This study claims that making the system kinematically redundant, by integrating a mechanism with lower inertial characteristics, can be used for operation time reduction with the proposed algorithms in this paper. Next section provides a general description of a redundant mechanism for planar tasks. Following this, algorithm developed for redundancy resolution in order to minimize the end-effector task completion time is presented. Algorithm is devised from the work of (Sartorio, 2004), which comprises designing the velocity profiles separately for two mechanisms to impose a desired joined velocity profile generation of end point.

\section{Planar Redundant Mechanism for Higher Acceleration Motion}

Planar redundant manipulator that is used in aimed trajectory generation algorithms is composed of two mechanisms that have independently controlled two DoFs in planar space. As illustrated in Fig. 1, the secondary mechanism, with relatively smaller workspace, is mounted onto the primary mechanism, which has lower acceleration capabilities due to its higher inertial properties. End-effector is assembled on the second mechanism, which will project the desired output $E(x, y)$ of motion as a result of unified motions of the two mechanisms.

Motion of the primary mechanism is indicated with capital $X$ and $Y$, while the secondary mechanism's motion occurs along $u$ and $v$ axes. The workspace location of $u$ and $v$ axes, which is shown in red in Fig. 1 depends on the position of $X$ and $Y$ axes. End-effector path in this case is defined with respect to the global coordinates which are $x$ - and $y$ - axes. It should be noted that, since the designated system is planned to be used in industrial applications, such as machining tools, 
the end effector velocity is limited for application type. For example, during operation, mounted tool will be most likely required to have relatively lower speed limit below the maximum achievable speed of mechanism for proper operation.

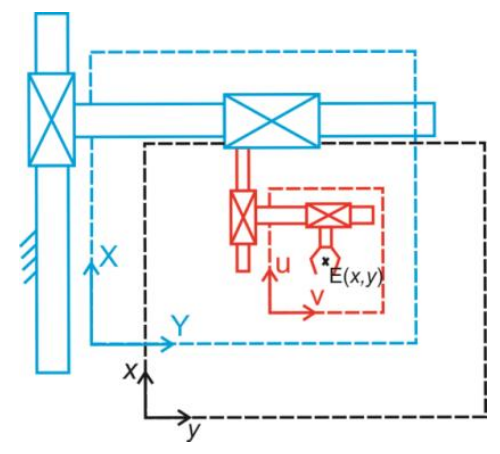

Fig. 1 Redundant mechanism.

\section{Trajectory Planning Algorithm}

Trajectory planning algorithm is developed to achieve the main task, which is position tracking of end-effector provided in global workspace. In general, the algorithm uses advantage of higher acceleration capabilities of secondary, low weight mechanism to reach maximum allowed velocity of end point, $E$, as quickly as possible. Unlike defining a main task and subtasks for redundant mechanisms, this algorithm comprises trajectory planning and control of two different mechanisms simultaneously. A possible solution to achieve a high acceleration trajectory planning algorithm for redundant manipulator can be determined in velocity profile generation level. In this algorithm, end-effector velocity profile is designed to have trapezoidal velocity profile with highest possible acceleration for endeffector as proposed in (Sartorio, 2004). Main aim is to shorten task completion duration compared to the conventional machine with only two axes.

The main function of the algorithm is to deploy end-effector position demands extracted from G-codes segments on redundant machining device (RMD) to utilize formerly mentioned capabilities. Trajectory planning algorithm is to be used as a built-in function in between G-code extractions of RMD and machine controller, which is typically a CNC system. The input data for motion planning are extracted from G-codes as segments in two categories: machining segments or travelling segments. In machining segments, the maximum speed of end-effector, or machining tool specifically, is limited by machining process type specifications. On the other hand, the travelling segments are created as positioning of tool before or in between machining segments with maximum motion capabilities of RMD. 
After the segments are created, the algorithm creates motion profiles sequentially with respect to the presented flowchart in Fig. 2. Segments data are fed as input to the motion planning scheme, which contain information about motion (distance travelled, start and end points of segment) and segment type. At first, the algorithm checks if there are any motions on the primary and secondary mechanisms from previous segments while the end-effector is kept at a constant position, which is further discussed in next sections. After that, if necessary, when acceleration adjustment is completed, the algorithm generates the velocity profile according to motion limitations for either travelling or machining segments.

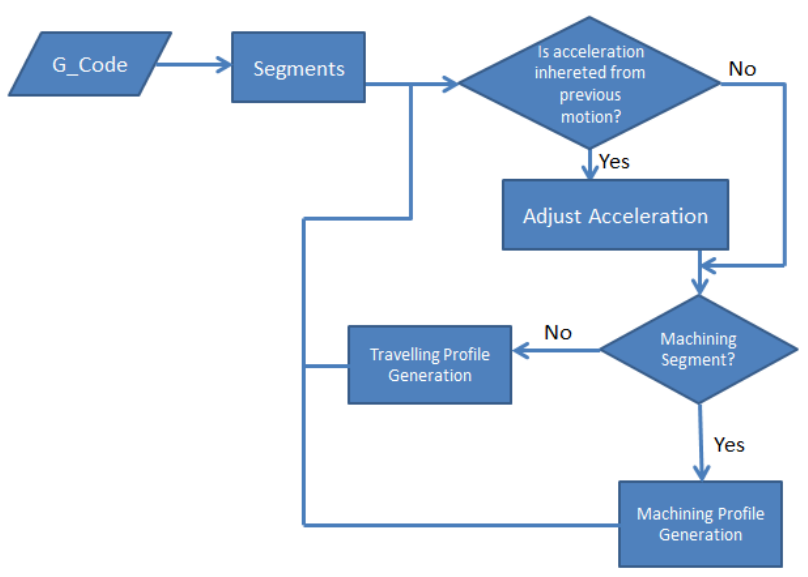

Fig. 2 Flowchart of trajectory planning algorithm.

In this study, the acceleration of the primary axes are designed to be limited with $9.81 \mathrm{~m} / \mathrm{s}^{2}$ for primary mechanism and $49.05 \mathrm{~m} / \mathrm{s}^{2}$ for secondary mechanism, and end-effector velocity is limited with $40 \mathrm{~m} / \mathrm{min}$. The maximum velocities are set $200 \mathrm{~m} / \mathrm{min}$ for primary mechanism, and $100 \mathrm{~m} / \mathrm{min}$ for secondary mechanism. Position demands delivered to secondary mechanism were held within workspace limitations as predesigned between $\pm 50 \mathrm{~mm}$ along both $\mathrm{u}$ and $\mathrm{v}$ axes.

\subsection{Velocity Profile Generation for Machining Segment}

A machining segment represents the motions that the end-effector follows during working on a workpiece. With redundancy resolution, this is achieved by adding two independently created velocity profiles for primary mechanism, $v_{a}$, along $X-Y$ axes and secondary mechanism, $v_{b}$, along $u-v$ axes to obtain the end-effector velocity profile. A continuous velocity profile, which is usually a small part Gcode sequence, is illustrated in Fig. 3. This velocity profile is applicable in cases where distance to be traveled is long enough to achieve proposed method. When distance is not enough to reach the maximum allowable velocity limits, a triangle 
velocity profile of the end-effector is used. Velocity of the end-effector is limited with $v_{e}(\max )$, which is the velocity limit for the designated machining process. Profile generation is designed such that secondary mechanism is designated to start motion at the center of its workspace, which is marked with $\mathbf{\square}$ (Fig. 1).

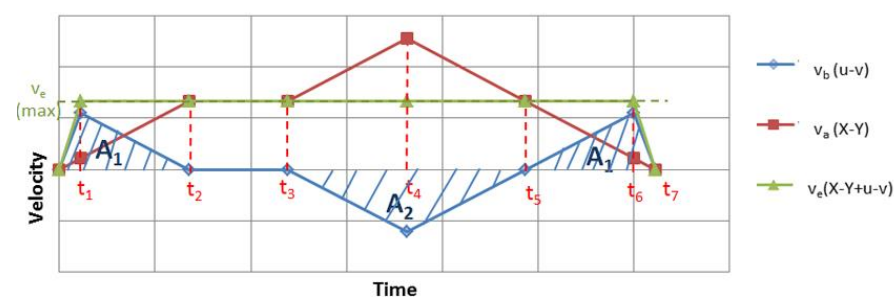

Fig. 3 Velocity level motion generation for machining segments.

After $v_{e}$ (max) is reached with combined accelerations of the two mechanisms, velocities of the two mechanisms are consistently regulated to preserve endeffector velocity at $v_{e}(\max )$ until time $t_{6}$. The duration that end-effector reaches its maximum velocity, $t_{l}$, is found by the combined acceleration of two mechanism which are primary axis limit, $a_{a}$, and secondary axis limit, $a_{b}$ (Equation 1).

$$
t_{1}=t_{7}-t_{6}=\frac{v_{e(\max )}}{a_{a}+a_{b}}
$$

Secondary mechanism, after $t_{l}$ seconds, decelerates at with a $-a_{a}$ value and comes to a full stop at $t_{2}$. After the primary mechanism reaches the $v_{e}(\max )$ at $t_{2}$, the velocity of both mechanisms are kept constant until $t_{3}$. Velocity of primary mechanism exceeds the end-effector velocity between $t_{3}$ and $t_{5}$ in order to retract secondary mechanism to the center of its workspace. Thus, when the total motion is completed, relative position of secondary mechanism will be at the center of its workspace to be ready for the next motion segment. Advantage of this algorithm is that the acceleration and deceleration are much higher at the beginning and termination of the motion, which shortens the total task completion duration.

Distance traveled by secondary mechanism as identified by $A_{1}$ and $A_{2}$ in Fig. 3 is calculated as presented in Equation 2, where $A_{2}=2 \times A_{1}$.

$$
A_{1}=1 / 2 \mathrm{a}_{\mathrm{b}} t_{1}^{2}+1 / 2 \mathrm{a}_{\mathrm{a}}\left(t_{2}-t_{1}\right)^{2} ; \quad A_{2}=1 / 2 \mathrm{a}_{\mathrm{b}}\left(t^{*}\right)^{2}
$$

$t^{*}$ is the duration between $t_{5}$ and $t_{3}$. which is calculated in Equation 3 .

$$
t^{*}=t_{1} \cdot \sqrt{1+\frac{a_{a}}{a_{b}}\left(\frac{a_{e}}{a_{a}}-1\right)^{2}}
$$

Time elapsed during constant velocity is calculated from required distance to be travelled, $d_{T}$, as represented in Equation 4 .

$$
\left(t_{3}-t_{2}\right)=\frac{d_{T}}{v_{e(\max )}}-\left(2 t_{2}+t_{1}+t^{*}\right)
$$

Motion planning of a machining segment with a traveling distance of $0.5 \mathrm{~m}$ for the end-effector is presented in Fig. 4. Motion is generated with respect to the described algorithm in this section for continuous motion of end-effector. According to calculations, the task is completed in $0.761 \mathrm{sec}$ with a maximum velocity of 40 
$\mathrm{m} / \mathrm{min}$. When compared with task completion duration $(0.818 \mathrm{sec})$ of the conventional mechanism, there is a $6.97 \%$ time gain. Although time reduction magnitudes for longer-distance machining are smaller, for a task with many smallerdistance contours, algorithm results in remarkably higher reductions in total.

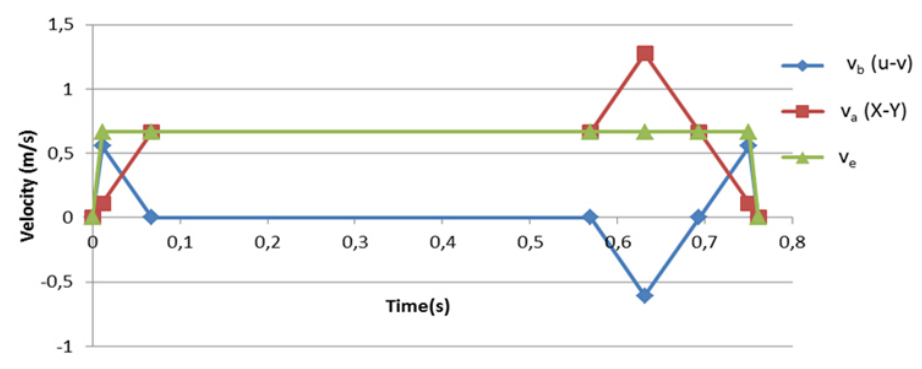

Fig. 4 Velocity level motion generation for $0.5 \mathrm{~m}$ machining distance.

\subsection{Velocity Profile Generation for Travelling Segment}

Travelling segments are reserved for motions generated for moving endeffector or machining tool to the desired initiation coordinates of the machining process. Travelling segments are planned between machining segments if there is an offset between termination and initiation coordinates of sequences of G-codes. Like in machining segment, travelling velocity profile generation is carried out for primary mechanism velocity, $v_{a}$, along $X-Y$ axes and secondary mechanism, $v_{b}$, along $u-v$ axes. In travelling velocity profiles, maximum acceleration duration and velocity generated by secondary mechanism is limited due to its workspace.

In Fig. 5, the velocity profiles generated for travelling segment are drawn according to primary and secondary mechanisms' velocity limits, $v_{a}(\max )$ and $v_{b}(\max ) \cdot v_{a}(\max )$ is set by primary mechanism's maximum velocity and $v_{b}(\max )$ is chosen to preserve secondary mechanism's motions inside its workspace. During $t_{0}-t_{l}$, secondary mechanism accelerates with maximum acceleration and decelerates with the same acceleration magnitude of the primary mechanism to stop at its workspace limit. As a result of this, the velocity of end-effector is kept constant while secondary mechanism decelerates and comes to a stop.

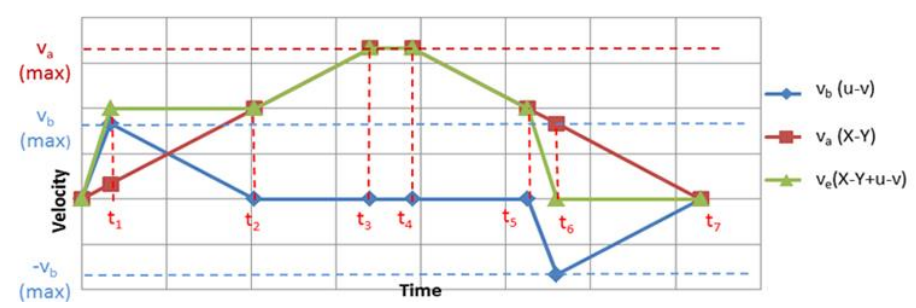

Fig. 5 Velocity level motion generation for travelling segment. 
Since at initiation and termination secondary mechanism should be located at it center point, distance travelled by end effector is calculated from motion of primary mechanism. Therefore, the $t_{3}$ and $t_{4}$ time values are determined by conventional trapezoidal velocity calculations using the maximum velocity and acceleration limits of primary mechanism and required distance to be travelled by end-effector.

The velocity limit of secondary axes $v_{b}(\max )$ is chosen to preserve secondary mechanism's motions inside its workspace. Thus, $t_{1}$ and duration between $t_{6}$ and $t_{5}$, is found by using the acceleration limit of the secondary mechanism $t_{l}=v_{b \max } / a_{b}$. $\mathrm{t}_{2}$ is calculated by Equation 5, which is also the equal to duration between $t_{7}$ and $t_{5}$.

$$
t_{2}=\frac{\left(\mathrm{a}_{\mathrm{b}}+\mathrm{a}_{\mathrm{a}}\right) t_{1}}{\mathrm{a}_{\mathrm{a}}}
$$

During the deceleration of end-effector, secondary mechanism moves in the reverse direction of the end-effector motion with its maximum acceleration initiating from the limit of its workspace. This results in a faster deceleration of the endeffector and the end-effector reaches the travelling segment's termination location. However, at $t_{6}$, while the end-effector completes its designated motion, the primary and secondary mechanisms still move. The motion on each mechanism from $t_{\sigma}$ to $t_{7}$ is described to hold the end-effector position constant while retracting the secondary mechanism to the center of its workspace. This is achieved by demanding $9,81 \mathrm{~m} / \mathrm{s}^{2}$ acceleration from both mechanisms in different directions. Primary mechanism velocity is designed to have a trapezoidal profile with maximum acceleration $\left(9.81 \mathrm{~m} / \mathrm{s}^{2}\right)$ and maximum speed $(200 \mathrm{~m} / \mathrm{min})$ between $t_{0}-t_{7}$. At the end of the motion described in Fig. 5, from $t_{6}$ to $t_{7}$, end-effector does not move and reaches its motion termination location. This may be seen as a loss of time. However, for any machining operation, there should be a time interval to cut into the workpiece and this dead time can be utilized for this purpose.

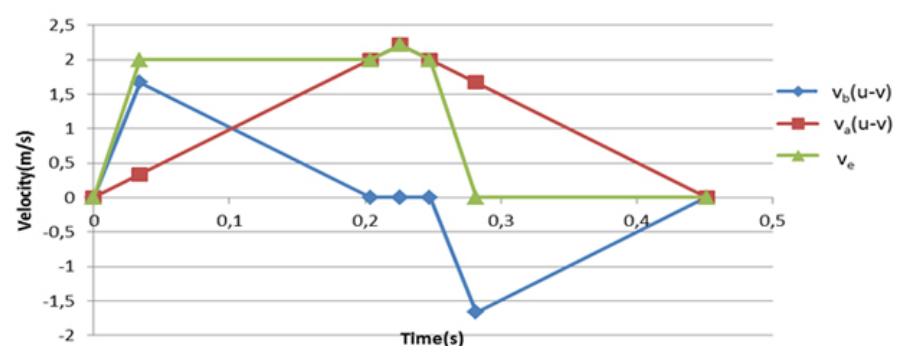

Fig. 6 Velocity level motion generation for $0.5 \mathrm{~m}$ travelling distance.

For the numerical example, motion planning of a travelling segment with a traveling distance of $0.5 \mathrm{~m}$ of the end-effector is presented in Fig. 6 . The calculations show that the travelling segment task is completed in $0.4515 \mathrm{sec}$ with a maximum velocity of $132.88 \mathrm{~m} / \mathrm{min}$ for primary mechanism. As it can be deduced from Fig. 6, there is $0.17 \mathrm{sec}$ of time gain (dead time) that can be used for initiation of the cutting process of tool. As a result, there will be time reduction from total completion of sequences of RMD's process. 


\section{Conclusion}

Conventional machines that have larger workspaces suffer in providing higher accelerations while preserving their precision specifications due to moving higher inertia components during the execution of the task. In this work, kinematic redundancy is proposed to enhance the acceleration capability for executing the same task with larger workspace limits. An example case study is carried out for planar motion. An algorithm is generated and discussed to distribute the global motion demand to the primary mechanism and secondary mechanism. Advantage of the algorithm is proved by numerical examples conducted for both machining and travelling segments. This algorithm also has an advantage with respect to pseudo-inverse methods that numerical calculation load is less since this method uses only simple algebraic calculations rather than dealing with matrix inversions. This method also guarantees that maximum allowable acceleration is achieved throughout the task to minimize total task execution duration. However, algorithm has a practical disadvantage that jerks are not infinite as utilized in this study. Thus, effects and compensation for these effects will be investigated.

\section{References}

Chen, J. L., Liu, J. S., Lee, W. C., Liang, T. C. (2002). On-line multi-criteria based collision-free posture generation of redundant manipulator in constrained workspace, Robotica, 20: 625636.

Guo, Z. Y., Hsia, T. C. (1990). Joint trajectory generation for redundant robots in an environment with obstacles. IEEE Inter. Conf. on Robotics and Automation, Cincinnati, OH, U.S.A, $157-162$.

Maaroof, O., Gezgin, E., Dede, M. İ. C. (2012). General subtask controller for redundant robot manipulators. 12. IEEE International Conference on Control, Automation, Jeju Island, Korea.

Nakamura, Y. (1991). Advanced Robotics Redundancy and Optimization, Addison-Wesley Pub. Co., Massachussetts, US.

Rajiv, V. D., James, A. E., Scott, M. (1991). Real-time implementation of an optimization scheme for seven-degree-of-freedom redundant manipulators. IEEE Transactions On Robotics And Automation, 1(5), 579-588.

Sartorio, F. (2004). Machine tool and manipulator devise adapted to be mounted on such machine. Patent No: US 2004/0025761 A1.

Seraji, H. (1991). Task options for redundancy resolution using configuration control, IEEE Conference on Decision and Control, Brighton, UK Vol. 3, 2793- 2798.

Tatlıcioğlu, E., Braganza, D., Burg, T. C., Dawson, D. M. (2009). Adaptive control of redundant robot manipulators with subtask objectives. Robotica, 27(6), 873-881.

Yoshikawa, T. (1984). Analysis and control of robot manipulators with redundancy, Proceedings of First International Sympossium on Robotics Research, Cambridge, MIT Press, 735-748.

Wang, J., Y. Li and X. Zhao (2010). Inverse kinematics and control of a 7-dof redundant manipulator based on the closed-loop algorithm, International Journal of Advanced Robotic Systems, 7(4), 1-9. 